\title{
Seismic Fragility Analysis of a Tank-Piping System based on Surrogate Modeling and Artificial Ground Motions
}

\author{
G. Abbiati ${ }^{\mathrm{a}}$, M. Broccardo ${ }^{\mathrm{b}}$, R. di Filippo ${ }^{\mathrm{b}}$, B. Stojadinovic ${ }^{\mathrm{c}}$, O.S. Bursi ${ }^{\mathrm{b}}$ \\ ${ }^{a}$ Department of Engineering, University of Aarhus, 8000 Aarhus, Denmark. \\ ${ }^{b}$ Department of Civil, Environmental and Mechanical Engineering, University of Trento, 38123 Trento, Italy. \\ ${ }^{c}$ Department of Civil, Environmental and Geomatic Engineering, ETH Zurich, 8192 Zurich, Switzerland.
}

\begin{abstract}
The catastrophic consequences of a few recent NaTech events have highlighted the inadequacy of the accepted approach to quantitative seismic risk assessments of chemical process plants. In particular, the systematic lack of system-level numerical simulations of the chemical process plant response to extreme seismic events is identified as a major pitfall. In response to that, a progressive integration of the PEER (Pacific Earthquake Engineering Research Center) Performance-Based Earthquake Engineering framework's best practices has been recently promoted. Fragility models are the key components of the framework. Specifically, a fragility model determines the probability of a plant component being in a damaged state conditional to a given intensity of seismic hazard and accounting for system-level interactions with other plant components. However, a handful of limitations still prevent a systematic implementation of the PEER framework to chemical process plants. The three most significant are a reduced number of hazard-consistent site-specific ground motion records for time history analyses, the computational cost of system-level simulations, the experimental cost for component-level model validation.

In response to these challenges, this paper proposes a recently developed uncertainty quantificationbased framework to perform seismic fragility assessments of chemical process plants. The framework employs three key elements: a stochastic ground-motion model to supplement the scarcity of real records, extensive surrogate modeling to reduce the computational cost of system-level simulations, and component-level model validation based on cost-effective hybrid tests.

The framework is applied to compute two fragility models for a pipe elbow of an ideal tank-piping system. The results show the great potential of the framework for fragility assessments of chemical
\end{abstract}

Email addresses: abbiati@eng.au.dk (G. Abbiati), marco.broccardo@unitn.it (M. Broccardo), rocco.difilippo@unitn.it (R.di Filippo), stojadinovic@ibk.baug.ethz.ch (B. Stojadinovic), oreste.bursi@unitn.it (O.S. Bursi) 
process plants.

Keywords: Seismic fragility analysis, PEER-PBEE framework, artificial ground motion, surrogate modeling, hybrid simulation, tank-piping system.

\section{Introduction}

2 Campedel [1] showed that natural hazards are the leading cause of $2 \pm 5 \%$ of Chemical Process Plants

3 (CPP) accidents. The study is based on an extensive review of historical data based on five European 4 and one US databases. Kidam and Hurme [2] found similar figures by analyzing 364 CPP accidents 5 from the Japanese Failure Knowledge Database. Such occurrences are classified as NaTech events, 6 that is, technological accidents triggered by natural disasters. Release of hazardous chemicals, fire, or explosions following a structural failure are often among the most significant consequences of 8 NaTech events [3, 4]. The Izmit refinery fire following the Kocaeli earthquake (Turkey, 1999) [5], 9 the leak of hazardous chemicals from multiple fertilizer plants following the Wenchuan earthquake 10 (China, 2008) [6], and the nuclear accident following the Fukushima earthquake-induced Tsunami 11 (Japan, 2011) [7] are just some examples of NaTech events associated with seismic hazard.

Their catastrophic consequences have highlighted the inadequacy of the accepted approach to Quantitative Risk Assessments (QRA) of CPPs [8]. In particular, the systematic lack of accurate ${ }_{14}$ system-level numerical simulations of the chemical process plant response to extreme seismic events

${ }^{1}$ Oftentimes damage analysis and fragility analysis are blended in one assessment 
supported by system-level numerical simulations of the CPP. In this case, the computation of fragility functions requires the selection of a set of suitable ground motions, which are used as input for nonlinear structural analysis [19, 20, 21]. Within this perimeter, Bradley [22] and Silva et. al. [23] collected a number of open issues. Although both studies refer to the seismic risk of civil structures, their conclusions are also valid for CPPs. Specifically, the authors highlighted the following three criticalities:

- Although methodologies for ground motion selection succeed in describing the seismic hazard for a given structure, the number of available records is usually too small for computing accurate QoIs' statistics.

- Experimental validation of structural simulators is often overlooked. This is due to practical reasons. Structural testing is usually not affordable beyond the component level due to the limited capacity of experimental facilities.

- The computation of fragility analysis requires a large number of time history analyses. It follows that computational expensive structural simulators limit de facto the total number of simulations.

Recently, Abbiati et al. [24] proposed a comprehensive framework that tackles these open challenges. This study adopts and applies this framework to compute the seismic fragility analysis of a tank-piping system of an ideal CPP. The building blocks of the computational framework are an artificial ground motion (AGM) model calibrated against hazard-compatible site-specific seismic records (e.g., the AGM model of Rezaian and Der Kiureghian in this study [25]), a high-fidelity (HF) structural simulator of the reference structure validated against experiments (e.g., hybrid simulation (HS) in the proposed application [26]), and a corresponding low-fidelity (LF) structural simulator (e.g., obtained via dynamic substructuring of the HF structural simulator [27]). The seismic fragility analysis follows these sequential steps: i) generation of an ensemble of AGMs compatible with the seismic hazard, ii) computation of a Polynomial Chaos Expansion (PCE) surrogate of the QoI prediction provided by the LF structural simulator, iii) calculation of PCE-based Sobol' sensitivity indices of the LF-based QoI w.r.t. the ground motion parameters, iv) dimensionality reduction of the parameter space of the AGM model, v) computation of a hierarchical kriging (HK) surrogate that fuses LF and HF realizations of the QoI, vi) fragility analysis computed via Monte-Carlo-based UQ forward analysis of the HK surrogate. 
59 analysis.

73 of the motion. This process can be written as follow

$$
A(t)=\mathcal{M}_{a}\left(t, Z \mid X_{a}\right)
$$

so Function $(\mathrm{CDF})$ of $Y$ given a realization $\boldsymbol{x}_{a}$ is simply

$$
F_{Y \mid \boldsymbol{X}_{a}}\left(y \mid \boldsymbol{x}_{\boldsymbol{a}}\right)=\mathbb{P}\left(\mathcal{M}_{s}\left(\boldsymbol{Z} \mid \boldsymbol{X}_{a}=\boldsymbol{x}_{a}\right) \leq y\right)=\int_{\boldsymbol{z}} \mathbb{I}\left(\mathcal{M}_{s}\left(\boldsymbol{z} \mid \boldsymbol{x}_{a}\right) \leq y\right) d F_{\boldsymbol{Z}}(\boldsymbol{z}),
$$

where $\mathbb{I}(\cdot)$ denotes the indicator function, $\mathbb{P}(\cdot)$ denotes probability, $F_{\boldsymbol{Z}}(\boldsymbol{z})$ denote the joint $\mathrm{CDF}$. 
82 Provided with $F_{Y \mid X_{a}}$ continuous and strictly monotonically increasing the $p$-quantile function is 83 simply $y_{p}=F_{Y \mid X_{a}}^{-1}\left(p \mid \boldsymbol{x}_{\boldsymbol{a}}\right)$. Observe that $y_{p}$ is a random quantity as it is function of $\boldsymbol{X}_{a}$. Therefore, ${ }_{84}$ we can formally introduce the random variable $Y_{p}$ as

$$
Y_{p}=F_{Y \mid X_{a}}^{-1}\left(p \mid \boldsymbol{X}_{a}\right)=\mathcal{M}_{p}\left(\boldsymbol{X}_{a}\right)
$$

where $\mathcal{M}_{p}(\cdot):=F_{Y \mid X_{a}}^{-1}(p \mid \cdot)$ is a deterministic model indexed by $p \in[0,1]$. In this framework, $Y_{p}$ (for different values of $p$ ) is the QoI used used for decision making.

In the proposed application, the computation of the statistics of $Y_{p}$ is a complex and expensive computational problem. In fact, $\mathcal{M}_{c}$ is a computationally expensive black-box finite-element solver and, therefore, the evaluation of $\mathcal{M}_{p}$ is costly too.

Provided with such a setting, a surrogate model replaces the original model

$$
\hat{Y}_{p}=\hat{\mathcal{M}}_{p}(\boldsymbol{X})
$$

where $\hat{Y}_{p} \approx Y_{p}$. The statistics of $\hat{Y}_{p}$ are, then, directly used for decision making. To define an optimal surrogate model w.r.t. the stochastic input, the computational solver, and QoI [24] designed a strategy that builds on PCE-based GSA and HK. Here, we summarize the main steps.

1. Model definition and validation. Define the QoI, the stochastic model of the input $\mathcal{M}_{a}$ (Section 4), and a set of structural simulators $\mathcal{M}_{c}$ (Section 3). In this application, $c \in$ $\{L F, H F\}$, where $L F$ stands for LF (state-space) structural simulator, and $H F$ for HF (finiteelement) structural simulator. The definition of the models follows the criteria reported in [24] and here synthesized

(a) The parameters of the stochastic model of the input are calibrated w.r.t. a hazard compatible site-specific seismic catalog.

(b) The HF model has passed a validation protocol. This study uses HF models validated against HS tests performed in the laboratory of the University of Trento, Italy.

(c) The LF model is a proxy of the HF model, which is computationally cheaper. In order to ensure the consistency required in a MF analysis ([24]), this study uses a LF model constructed via dynamic substructuring of the HF model [27].

2. Initial experimental design. Create a large Experimental Design (ED) $\left\{\boldsymbol{y}_{L F, p}, \boldsymbol{X}_{a}\right\}$, where $\boldsymbol{y}_{L F, p}=\left\{y_{L F, p}^{(1)}, \ldots, y_{L F, p}^{\left(N_{L F}\right)}\right\}, \boldsymbol{X}_{a}=\left\{\boldsymbol{x}_{a}^{(1)}, \ldots, \boldsymbol{x}_{a}^{\left(N_{L F}\right)}\right\}$, and $\boldsymbol{x}_{a}^{(n)}$ is a generic sample of the input parameters, and $y_{L F, p}^{(n)}$ is the corresponding LF estimate. 
3. PC-based global sensitivity analysis. Train a non-intrusive PCE surrogate model, $\hat{\mathcal{M}}_{L F, p}^{P C E}\left(\boldsymbol{x}_{a}\right)$, using $\left\{\boldsymbol{y}_{L F, p}, \boldsymbol{X}_{a}\right\}$, and perform a PCE-based GSA [28]. Retain the input random variables that contribute most to the variability (variance) of the QoI and fix the remaining ones to their expected value.

4. Refined experimental design. Train a reduced $\operatorname{ED}\left\{\boldsymbol{y}_{H F, p}, \boldsymbol{X}_{\alpha}\right\}$, where $\boldsymbol{y}_{H F, p}=\left\{y_{H F, p}^{(1)}\right.$, $\left.\ldots, y_{H F, p}^{\left(N_{H F}\right)}\right\}, \boldsymbol{X}_{\alpha}=\left\{\boldsymbol{x}_{\alpha}^{(1)}, \ldots, \boldsymbol{x}_{\alpha}^{\left(N_{H F}\right)}\right\}$, and $\boldsymbol{x}_{\alpha}^{(n)}$ is a generic samples of the "important" model parameters, and $\boldsymbol{X}_{\alpha}$ the collection of such samples.

5. Kriging MF surrogate modelling. Train a MF Kriging surrogate $\hat{\mathcal{M}}_{M F}^{K}\left(\boldsymbol{x}_{\alpha}\right)$ based on $\left\{\boldsymbol{y}_{H F, p}, \boldsymbol{X}_{\alpha}\right\}$, which uses the PCE surrogate as trend function.

6. Probabilistic characterization of the QoI. Compute the statistics of the QoI of interest via forward Monte-Carlo-based UQ analysis of the MF surrogate model.

In a Performed Based Design setting, the QoI, $Y_{p}$, is usually an $E D P$. It follows that this framework allows the computation of any statistic of interest for the selected $E D P$, including fragility functions and surfaces w.r.t. any input variable included in $\boldsymbol{X}_{a}$.

\section{Tank-piping system case study}

The tank-piping system represents the portion of an ideal CPP located in Hanford, California. As depicted in Figure 1, the piping system is connected to a slender steel tank through a bolted flange joint. In detail, the piping system comprises 8" (outer diameter: $219.08 \mathrm{~mm}$; thickness: $8.18 \mathrm{~mm}$ ) and 6" (outer diameter: $168.28 \mathrm{~mm}$; thickness: $7.11 \mathrm{~mm}$ ) tubes, two 90 deg elbows, one bolted flange joint and one tee joint. The steel tank is characterized by radius $R=4.00 \mathrm{~m}$, height $H=14.00 \mathrm{~m}$ and thickness $T=0.006 \mathrm{~m}$, and it is filled with a fluid of $900.00 \mathrm{~kg} / \mathrm{m}^{3}$ density. The system is subjected to a ground motion acceleration parallel to the main axis of the piping.

In line with the presented framework, two structural simulators of the tank-piping system, characterized by different degrees of fidelity, were implemented. Both models assumed a linear elastic behavior for the piping system, which was discretized using 2D Euler-Bernoulli beam elements. Straight linear elastic 2D Euler-Bernoulli beams were also used for the elbow in the LF structural simulator, which was implemented in MATLAB. Joints and layouts significantly influence the overall piping vulnerability, as shown in different studies [29, 30, 18]. In particular, the occurrence of leakage is highly correlated to stress concentrations in pipe bends, as highlighted in [17]. Hence, 3D shell-based elbow elements endowed with an elastoplastic constitutive law with kinematic hardening 


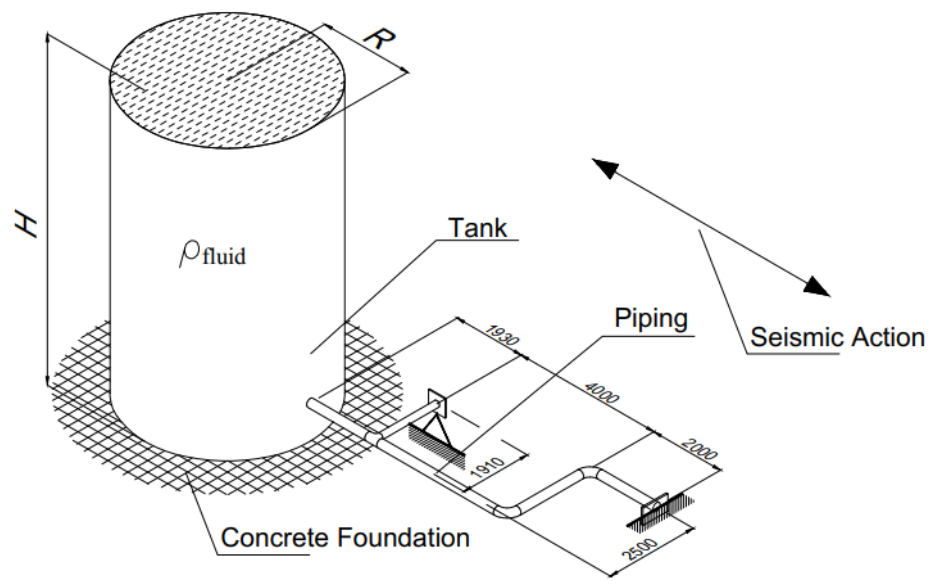

Figure 1: Tank-piping system case study.

${ }_{139}$ were used for the HF structural simulator. For a review of computational modeling issues related to

140 piping bends, the reader is addressed to [31].

${ }_{141}$ Both LF and HF structural simulators rely on the same simplified 3-DoFs tank model whose formulation is reported in [32]. An elastoplastic spring was used to model the Coulomb friction activated by tank sliding. The related cut-off force was computed based on the weight of the tank filled and a friction coefficient $\mu=0.1$, activated by a yield displacement of $1 \mathrm{~mm}$. Table 1 summarizes the tank parameter values (i.e., $\boldsymbol{x}_{c}$ ). A COMBIN40 element was used to implement the elastoplastic spring in the HF structural simulator in ANSYS, whereas the bilinear elastoplastic spring described in [33] was used for the LF structural simulator implemented in MATLAB.

148 Figure 2 a reports a schematic of the LF structural simulator of the tank-piping system, whereas

Figure 2 reports the stress-strain relationship of the material constitutive law adopted for the steel of HF elbows.

The computational time needed for solving a single time history response analysis of the LF structural simulator was about $10 \mathrm{~s}$. In contrast, a single evaluation of the time history response of the $\mathrm{HF}$ structural simulator was about $500 \mathrm{~s}$. In detail, both cases refer to a $15 \mathrm{~s}$ long accelerogram using a standard laptop with an Intel $1.80 \mathrm{GHz}$ i7-8565U CPU and 16 GB RAM. 


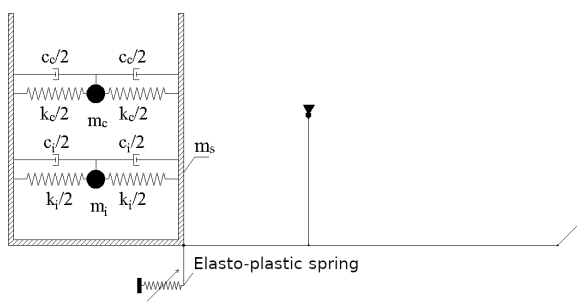

(a)

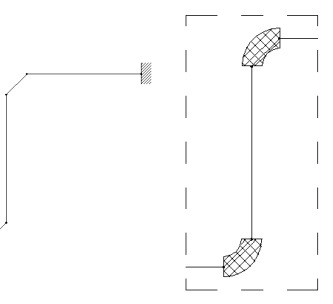

(b)

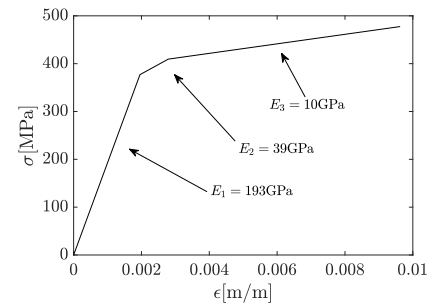

(c)

Figure 2: Computational modeling of the tank-piping system: a) schematic of the LF structural simulator; b) 3D shell-based elbow elements of the HF structural simulator; c) stress-strain relationship of the steel constitutive law adopted in the HF elbows.

\section{Stochastic modeling of the seismic input}

In this study, the AGM model proposed by Rezaian and Der Kiureghian [25, 34] was selected to represent the seismic input for the fragility analysis. This AGM model consists of a time-modulated band-limited non-stationary white noise described by the following equation,

$$
A(t)=\mathcal{M}_{a}\left(t, Z \mid \boldsymbol{X}_{a}\right)=q\left(t \mid \boldsymbol{X}_{q}\right) \frac{h\left(t \mid \boldsymbol{X}_{h}\right) * W(t, \boldsymbol{Z})}{\sigma_{h}\left(t, \boldsymbol{Z} \mid \boldsymbol{X}_{h}\right)}
$$

where $*$ denotes time convolution; $\boldsymbol{X}_{a}=\left[\boldsymbol{X}_{q}, \boldsymbol{X}_{h}\right] ; q\left(t \mid \boldsymbol{X}_{q}\right)$ is a parametric time modulating function with random parameters $\boldsymbol{X}_{q} ; h\left(t \mid \boldsymbol{X}_{h}\right)$ is the impulse-response function of a linear filter with time varying random parameters, where $\boldsymbol{X}_{h}$ represents a set of time invariant parameters; $W(t, Z)=\sum_{n}^{N_{t}} \delta\left(t-t_{n}\right) Z_{n}$ is a band-limited white noise process, where $Z=\left[Z_{1}, \ldots Z_{N_{t}}\right]$ is a standard normal Gaussian vector; and $\sigma_{h}\left(t, Z \mid \boldsymbol{X}_{h}\right)$ is the variance of the convoluted process up to time $t$. The modulating function proposed is,

$$
q\left(t \mid \boldsymbol{X}_{q}\right)=\alpha_{1} t^{\alpha_{2}-1} \exp \left(-\alpha_{3} t\right), \text { if } t>0,0 \text { elsewhere }
$$

where $\boldsymbol{X}_{q}=\left[\alpha_{1}, \alpha_{2}, \alpha_{3}\right]$ are the parameters of the function. Moreover, it can be shown that a one-to-one mapping exists between these parameters and $D_{5-95}=T_{95}-T_{5}, T_{45}$, and $I_{a}$ - where $T_{p}$ is the time corresponding to the $p \%$ of the cumulative Arias intensity of the residual, $I_{a, r e s}$. It follows that $\boldsymbol{X}_{q}$ can be written as $\boldsymbol{X}_{q}=\left[I_{a, \text { res }}, T_{45}, D_{5-95}\right]$. The time-varying filter is defined by

$$
h\left(t \mid \boldsymbol{X}_{h}\right)=\frac{\omega_{f}(t)}{\sqrt{1-\zeta_{f}^{2}}} \exp \left[-\omega_{f}(t) \zeta_{f} \cdot t\right] \sin \left[\omega_{f}(t) \sqrt{1-\zeta_{f}^{2}} \cdot t\right]
$$


Table 1: Simplified tank model parameters.

\begin{tabular}{clrl}
\hline Parameter $\boldsymbol{x}_{c}$ & Description & Value & Units \\
\hline$m_{s}$ & Sliding mass & 22.91 & ton \\
$m_{c}$ & Convective mass & 79.80 & ton \\
$m_{i}$ & Impulsive mass & 547.00 & ton \\
$k_{c}$ & Convective stiffness & 359 & $\mathrm{~N} / \mathrm{mm}$ \\
$k_{i}$ & Impulsive stiffness & 685810 & $\mathrm{~N} / \mathrm{mm}$ \\
$\omega_{c}$ & Convective frequency & 2.12 & $\mathrm{rad} / \mathrm{s}$ \\
$\omega_{i}$ & Impulsive frequency & 35.40 & $\mathrm{rad} / \mathrm{s}$ \\
$\zeta_{c}$ & Convective damping & 0.005 & - \\
$\zeta_{i}$ & Impulsive damping & 0.05 & - \\
\hline
\end{tabular}

In Eq. (7), the main frequency, $\omega_{f}(t)$, evolves linearly with time. Specifically, $\omega_{f}(t)=\omega_{\text {mid }}+$ $\dot{\omega}\left(t-T_{45}\right)$, where $\omega_{\text {mid }}$ is the frequency at time $T_{45}$ and $\dot{\omega}$ is the rate of change of the frequency with time. The damping of the filter, $\zeta_{f}$, is considered time invariant. It follows that $\boldsymbol{X}_{h}=\left[\omega_{\text {mid }}, \dot{\omega}, \zeta\right]$.

Unlike the original work [25, 34], AGM model parameters were calibrated for each real record of a selected pool instead of using regression analysis with respect to seismic hazard characteristics. In detail, based on the disaggregation of the seismic hazard corresponding to PGA with probability of exceedance of $2 \%$ in 50 years, the seven ground motion records of the Northridge earthquake (California, 1994) reported in Table 2 were selected from the NGA database [35]. For both principal components of the selected ground motions, which were extracted according to the procedure described in [36], the AGM parameters were calibrated following the procedure reported in [34]. In this regard, Table 3 summarizes the obtained ranges of AGM model parameters.

Two sets of 4 and 3 artificial ground motions were selected to investigate system response at Serviceability Limit State (SLS) and the Ultimate Limit State (ULS), respectively, using HS. Specifically, based on nonlinear response analysis of the LF structural simulator, SLS accelerograms were selected to limit tank sliding peak to $0.04 \mathrm{~m}$ so that the piping response remained in the linear regime. On the other hand, ULS artificial accelerograms were selected to ensure a minimum tank sliding peak of $0.06 \mathrm{~m}$, thus causing the yielding of the elbow elements. Figure 3 depicts acceleration, velocity, and displacement response spectra of both the real records and the artificial ground motions 
used for HS. The results of the HS campaign are discussed in Section 5

Table 2: Selected records of the 1994 Northridge earthquake.

\begin{tabular}{lcc}
\hline Station Name & Moment magnitude $\left[\mathrm{M}_{\mathrm{w}}\right]$ & Epicentral distance $[\mathrm{km}]$ \\
\hline Canoga Park - Topanga Can & 6.69 & 14.70 \\
Canyon Country - W Lost Cany & 6.69 & 12.44 \\
N Hollywood - Coldwater Can & 6.69 & 12.51 \\
Northridge - 17645 Saticoy St & 6.69 & 12.09 \\
Simi Valley - Katherine Rd & 6.69 & 13.42 \\
Sun Valley - Roscoe Blvd & 6.69 & 10.05 \\
Sunland - Mt Gleason Ave & 6.69 & 13.35 \\
Pacoima Kagel Canyon & 6.05 & 11.34 \\
\hline
\end{tabular}

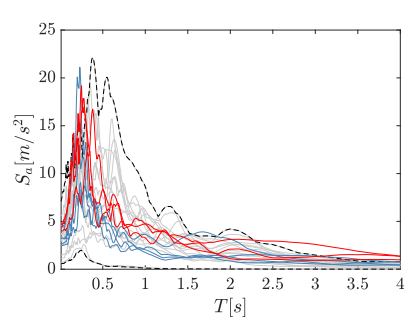

(a)

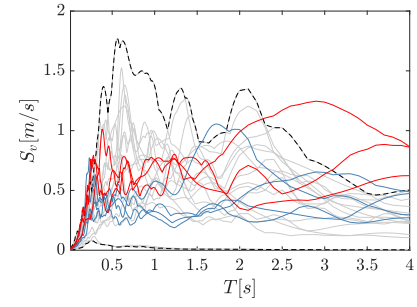

(b)

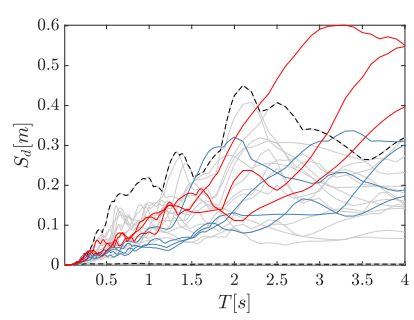

(c)

Figure 3: Selected records of the 1994 Northridge earthquake: a) acceleration; b) velocity; c) displacement response spectra. Grey lines refer to single retained records, whereas black dashed lines indicate the corresponding $95 \%$ confidence interval. Blue and red lines indicate SLS and ULS records produced with the calibrated AGM and used for the HS campaign, respectively.

\section{Model validation based on hybrid simulations}

To support the validation of the HF finite-element model of the piping system, a HS campaign was performed at the University of Trento, Italy. In details, the simplified model of the sliding tank (Section 3) constitutes the numerical substructure, and the piping system filled with 15 bar pressured water is the physical substructure. Figure 4 provides an overview of the experimental setup. Specifically, a hydraulic actuator of $250 \mathrm{kN}$ force capacity and $\pm 250 \mathrm{~mm}$ stroke was used 
Table 3: Intervals of the AGM model parameters.

\begin{tabular}{cccc}
\hline Name & Lower bound & Upper bound & Units \\
\hline$I_{a}$ & 1.019 & 3.992 & $\mathrm{~m}^{2} / \mathrm{s}^{3}$ \\
$D_{5-95}$ & 5.083 & 16.810 & $\mathrm{~s}$ \\
$T_{45}$ & 1.596 & 5.664 & $\mathrm{~s}$ \\
$\omega_{\text {mid }}$ & 14.620 & 31.000 & $\mathrm{rad} / \mathrm{s}$ \\
$\zeta$ & 0.074 & 0.557 & - \\
\hline
\end{tabular}

to test the piping in displacement-control mode. A comprehensive description of the HS algorithm used in this experimental campaign is reported in [37]. As thoroughly discussed in [17], hoop strain is an effective indicator of the damage level experienced by pipe elbows. Accordingly, a set strain gauges measured the hoop strain of each elbow, as depicted in Figure 5.

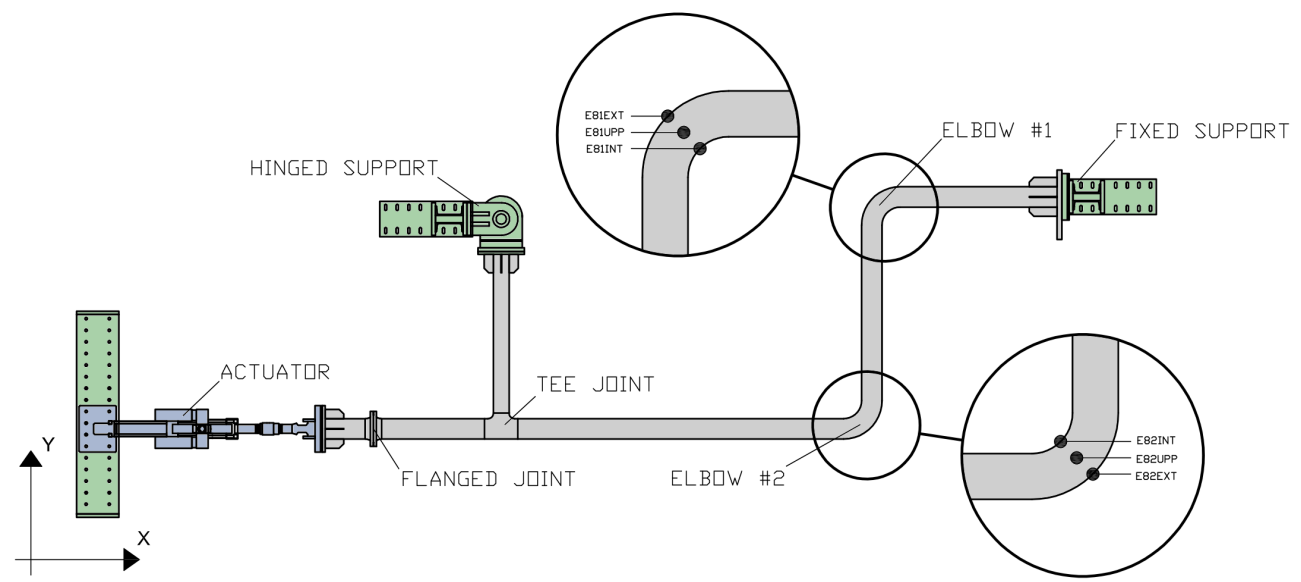

Figure 4: Schematic of the experimental setup. Labels E81INT, E81EXT and E81UPP indicate the strain gauges measuring the hoop strain of Elbow \#1 at three different locations. Simularly, E82INT, E82EXT and E82UPP refer to Elbow \#2.

As anticipated in Section 4, seven HSs were performed considering four artificial accelerograms for the SLS and three artificial accelerograms for the ULS. For the sake of simplicity, HS experiments are referred to as SLS/ULS \#i to specify the accelerogram used for the test. In all cases, experiments were conducted in the so-called pseudodynamic regime considering a testing time scale equal to 64 . Figures 6 and 7 provide an overview of the time history response of the piping system obtained via 


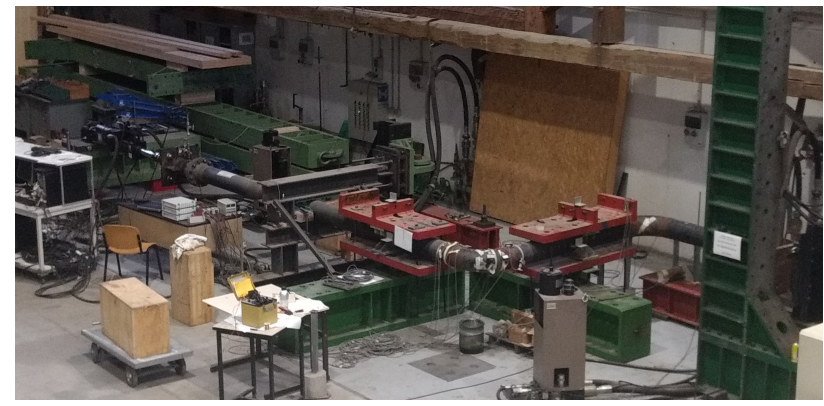

(a)

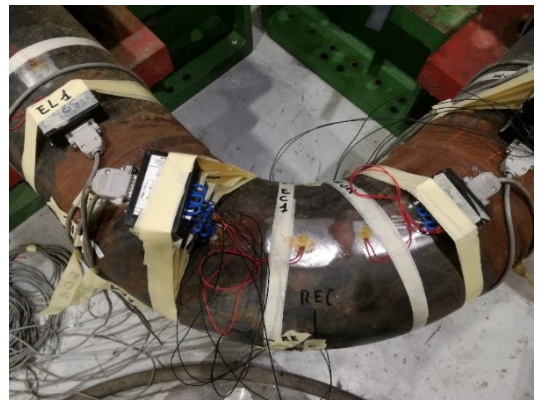

(b)

Figure 5: Experimental setup: a) overview of the installation at the Laboratory of Structures and Materials of the University of Trento, Italy; b) close-up view of Elbow \#2 instrumented with strain gauges.

HS for SLS and ULS artificial accelerograms, respectively. Figure 6 shows that the piping system response remained in the linear regime with SLS accelerograms. In this case, one can observe a slight hysteresis characterizing the restoring force. This is possibly due to the friction between the piping and the Teflon support pads. On the other hand, with reference to ULS accelerograms, Figure 7 shows that the response of the piping exceeded the linear regime. This observation is also confirmed by the measured strain histories.

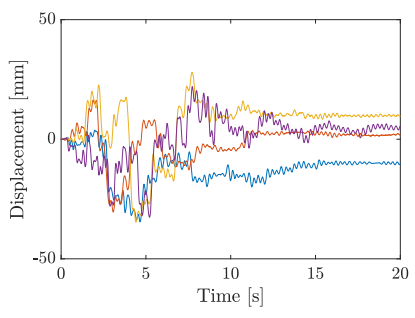

(a)

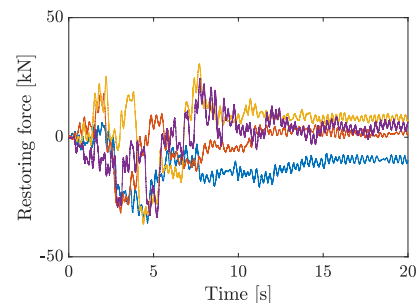

(b)

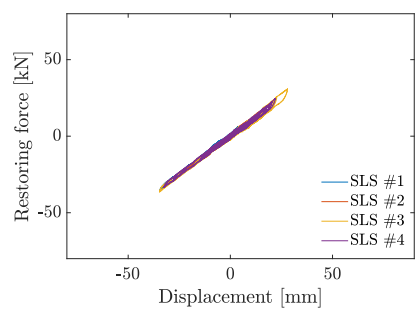

(c)

Figure 6: Experimental response of the piping obtained via HS using SLS accelerograms: a) displacement history; b) restoring force history; c) displacement vs. restoring force loop measured at the actuation point.

The experimental response of the piping was used to calibrate the HF structural simulator, and in particular, the constitutive model for the steel material of elbow elements. In this regard, Figure 8 compares the hoop strain histories of Elbow \#1 measured during HS to both LF and HF structural simulator predictions. Figure 8 shows that the validated HF structural simulator of the piping system reproduces the experimental measurement accurately. On the other hand, a bias is observed in the LF prediction. The latter, however, effectively reproduces the time modulation of the hoop strain. It 


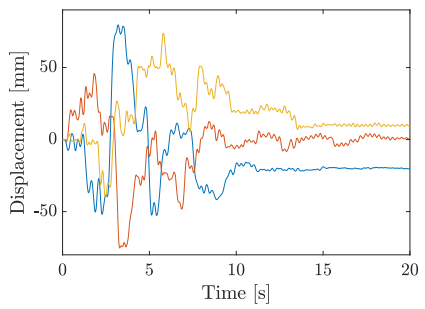

(a)

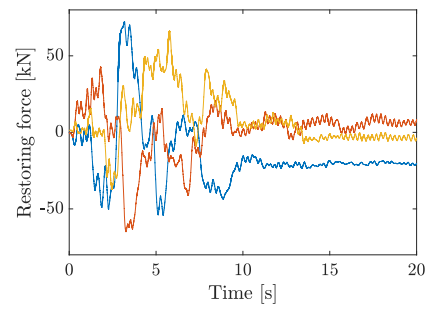

(b)

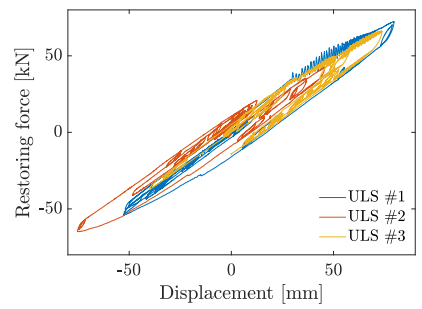

(c)

Figure 7: Experimental response of the piping obtained via HS using ULS accelerograms: a) displacement history; b) restoring force history; c) displacement vs. restoring force loop measured at the actuation point. $222-0.568 \mathrm{rad} / \mathrm{s}^{2}$. HF simulations. read

$$
\boldsymbol{Y}=\epsilon_{1}, \boldsymbol{X} \equiv \boldsymbol{X}_{a}=\left\{I_{a}, D_{5-95}, T_{45}, \omega_{m i d}, \zeta\right\}
$$

Figure 8: Hoop strain history of Elbow \#1 measured by sensor E81UPP during the HS ULS \#2 and corresponding LF and

\section{Fragility analysis of the tank-piping system}

Provided with the notation introduced in this study, input variables and QoIs for the fragility analysis 
According to step 2 of the proposed framework, an initial ED of 200 samples was evaluated using the LF structural simulator and considering QoI quantiles $p=\{0.05: 0.05: 0.95\}$. To ensure stable quantile estimates up to $95 \%, 100$ realizations of the AGM were evaluated for each point of the ED for a total of 20,000 LF simulations. Then, PCE-based Sobol' sensitivity indices were computed. Tables 4 and 5 report PCE-based first-order and total Sobol' sensitivity indices related to Elbow \#1. The contribution of $I_{a}, \omega_{\text {mid }}$ and $\zeta$ to the variability of Elbow \#1 QoI is dominant for all quantiles. Therefore, they were retained as input variables for the subsequent HK surrogate modeling phase. The remaining parameters of the AGM were set to constant average values $D_{5-95}=10.44 \mathrm{~s}$ and $T_{45}=3.70 \mathrm{~s}$. For the sake of space, only quantiles $p=\{0.1: 0.1: 0.9\}$ are reported. Tables 4 and 5 report also leave-one-out errors $\varepsilon_{\text {loo }}$ to report the accuracy of PCE surrogates. In the case of regression-based PCE, $\varepsilon_{\text {loo }}$ can be calculated directly from the coefficients of the PCE computed with the entire ED [38].

Table 4: First-order PCE-based Sobol' indices of the hoop strain peak of Elbow \#1 (QoI).

\begin{tabular}{|c|c|c|c|c|c|c|}
\hline$p$ & $\varepsilon_{\text {loo }}$ & $I_{a}$ & $D_{5-95}$ & $T_{45}$ & $\omega_{\text {mid }}$ & $\zeta$ \\
\hline 0.000 & 0.036 & 0.750 & 0.016 & 0.000 & 0.102 & 0.050 \\
\hline 0.100 & 0.006 & 0.690 & 0.023 & 0.004 & 0.112 & 0.083 \\
\hline 0.200 & 0.004 & 0.678 & 0.028 & 0.004 & 0.107 & 0.093 \\
\hline 0.300 & 0.004 & 0.668 & 0.029 & 0.005 & 0.114 & 0.094 \\
\hline 0.400 & 0.004 & 0.656 & 0.030 & 0.006 & 0.114 & 0.101 \\
\hline 0.500 & 0.005 & 0.641 & 0.032 & 0.008 & 0.117 & 0.108 \\
\hline 0.600 & 0.004 & 0.630 & 0.034 & 0.009 & 0.118 & 0.112 \\
\hline 0.700 & 0.004 & 0.616 & 0.039 & 0.011 & 0.119 & 0.117 \\
\hline 0.800 & 0.005 & 0.612 & 0.036 & 0.013 & 0.117 & 0.123 \\
\hline 0.900 & 0.007 & 0.596 & 0.036 & 0.016 & 0.119 & 0.131 \\
\hline
\end{tabular}

5 Among retained parameters, first-order summands of Figure 9 highlight monotonic increasing relationships with the QoI only for $I_{a}$ and $\zeta$. Hence, they were employed as IMs for computing the fragility analysis.

According to step 3 of the computational framework, a refined ED of 20 samples was evaluated using the HF structural simulator considering, 
Table 5: Total PCE-based Sobol' indices of the hoop strain peak of Elbow \#1 (QoI).

\begin{tabular}{|c|c|c|c|c|c|c|}
\hline$p$ & $\varepsilon_{\text {loo }}$ & $I_{a}$ & $D_{5-95}$ & $T_{45}$ & $\omega_{\text {mid }}$ & $\zeta$ \\
\hline 0.000 & 0.036 & 0.823 & 0.030 & 0.010 & 0.145 & 0.084 \\
\hline 0.100 & 0.006 & 0.772 & 0.034 & 0.005 & 0.157 & 0.122 \\
\hline 0.200 & 0.004 & 0.763 & 0.041 & 0.006 & 0.148 & 0.138 \\
\hline 0.300 & 0.004 & 0.751 & 0.044 & 0.007 & 0.152 & 0.137 \\
\hline 0.400 & 0.004 & 0.740 & 0.045 & 0.009 & 0.152 & 0.148 \\
\hline 0.500 & 0.005 & 0.727 & 0.047 & 0.011 & 0.156 & 0.157 \\
\hline 0.600 & 0.004 & 0.718 & 0.050 & 0.012 & 0.157 & 0.163 \\
\hline 0.700 & 0.004 & 0.703 & 0.056 & 0.014 & 0.158 & 0.169 \\
\hline 0.800 & 0.005 & 0.698 & 0.055 & 0.019 & 0.156 & 0.173 \\
\hline 0.900 & 0.007 & 0.683 & 0.055 & 0.026 & 0.156 & 0.184 \\
\hline
\end{tabular}

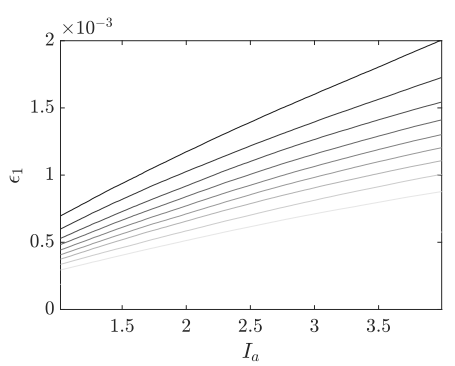

(a)

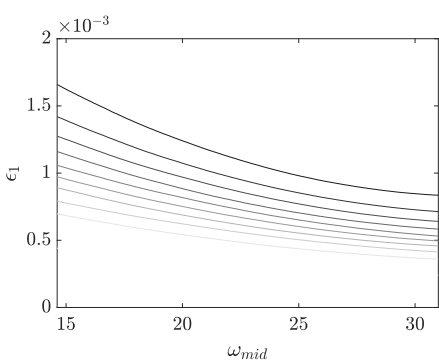

(b)

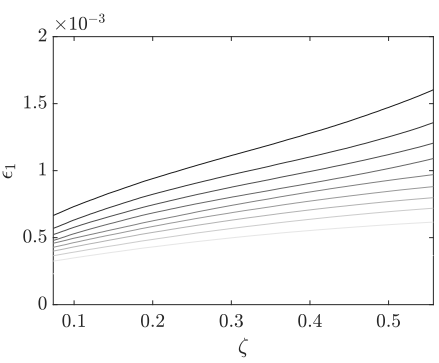

(c)

Figure 9: First-order summands of the hoop strain peak of Elbow \#1 (QoI) for the quantiles reported in Tables 4 and 5 Lighter curves refer to quantile $p=0.1$ while darker curves refer to quantile $p=0.9$.

$$
\boldsymbol{X}_{\alpha}=\left\{I_{a}, \omega_{\text {mid }}, \zeta\right\}, \boldsymbol{x}_{\beta}=E\left[\left\{T_{45}, D_{5-95}\right\}\right]
$$

For each sample of the refined ED, 100 AGM realizations were evaluated for a total of 2,000 HF simulations. Then, HK surrogates were trained for different ED sizes. Accordingly, HK- $n$ indicates a HK surrogate computed with $n$ samples of the HF structural simulator response quantiles. The PCE surrogate trained on the LF simulator was used as a trend function for the HK surrogates. A Matérn $5 / 2$ correlation function $R\left(\boldsymbol{X}-\boldsymbol{X}^{\prime} \mid \boldsymbol{\theta}\right)$ and second-order polynomial trend function $\boldsymbol{\mu}(\boldsymbol{X})$ were adopted. 


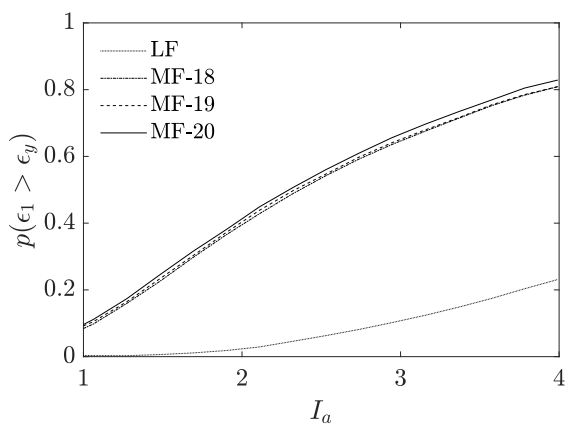

(a)

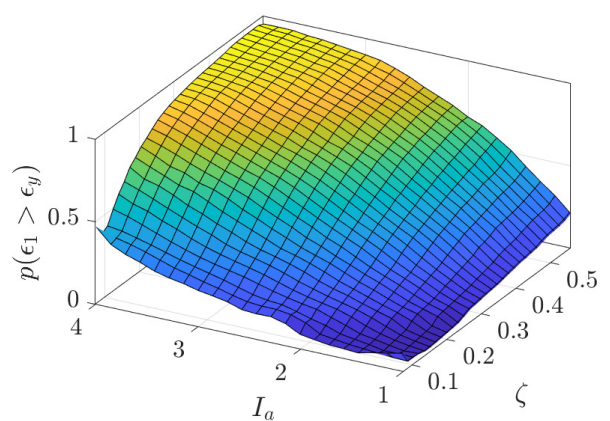

(b)
Figure 10: Fragility models for yielding limit state of Elbow \#1 assuming a hoop strain threshold value $\epsilon_{y}=1.70 \times 10^{-3}:$ a) fragility curve; b) fragility surface.

Although both LF and HF EDs assumed independent uniformly distributed AGM input parameters, 
hazard. Also, AGM input parameters can be mapped to instrumental IM, as illustrated in [40].

\section{Conclusion}

This study presented the application to chemical process plant components of a novel computational framework for fragility analysis. The framework addresses the following criticalities associated with the current fragility analysis practice: i- scarcity of hazard-specific site-consistent ground motion records, ii- high computational cost of high-fidelity simulations, iii- high experimental cost of model validation. In detail, the framework uses i- a stochastic ground motion model to augment the number of time series, ii- extensive use of surrogate and multi-fidelity modeling to drastically reduce the computational cost, iii- hybrid simulation tests to validate the computational models at a reduced experimental cost. The case study consisted of a tank-piping system of an ideal chemical process plant. Fragility models were computed for one of the two elbows of the piping system using a biased yet computational cheap low-fidelity simulator and an expensive-to-evaluate high-fidelity simulator that was validated against experiments. The same stochastic ground motion model was used for producing artificial records both for hybrid simulation and fragility analysis. The results highlight the great flexibility and potential of the method for a fragility assessment of chemical process plant components.

\section{Acknowledgements}

The authors wish to acknowledge Dr. Stefano Marelli (Chair of Risk, Safety and Uncertainty Quantification, ETH Zurich) and Dr. Imad Abdallah (Chair of Structural Mechanics, ETH Zurich) for providing constructive comments during the preparation of this manuscript. Also, the EU SERA project (Grant No. 730900) is acknowledged. Finally, the second, the third, and the last authors were supported by the Italian Ministry of Education, University and Research (MIUR) in the frame of the "Departments of Excellence" (grant L 232/2016).

\section{References}

[1] M. Campedel, Analysis of Major Industrial Accidents Triggered by Natural Events Reported In the Principal Available Chemical Accident Databases, Tech. rep.

[2] K. Kidam, M. Hurme, Analysis of equipment failures as contributors to chemical process accidents, Process Safety and Environmental Protection 91 (1-2) (2013) 61-78. 
[3] A. M. Cruz, L. J. Steinberg, A. L. Vetere-Arellano, Emerging Issues for Natech Disaster Risk Management in Europe, Journal of Risk Research 9 (5) (2006) 483-501.

[4] L. J. Steinberg, H. Sengul, A. M. Cruz, Natech risk and management: an assessment of the state of the art, Natural Hazards 46 (2) (2008) 143-152.

[5] H. Sezen, A. S. Whittaker, Seismic Performance of Industrial Facilities Affected by the 1999 Turkey Earthquake, Journal of Performance of Constructed Facilities 20 (1) (2006) 28-36.

[6] E. Krausmann, A. M. Cruz, B. Affeltranger, The impact of the 12 May 2008 Wenchuan earthquake on industrial facilities, Journal of Loss Prevention in the Process Industries 23 (2) (2010) 242-248.

[7] P. Y. Lipscy, K. E. Kushida, T. Incerti, The Fukushima Disaster and Japan's Nuclear Plant Vulnerability in Comparative Perspective, Environmental Science \& Technology 47 (12) (2013) 6082-6088.

[8] S. Esposito, B. Stojadinović, A. Babič, M. Dolšek, S. Iqbal, J. Selva, M. Broccardo, A. Mignan, D. Giardini, Risk-Based Multilevel Methodology to Stress Test Critical Infrastructure Systems, Journal of Infrastructure Systems 26 (1) (2020) 04019035.

[9] A. C. Caputo, F. Paolacci, O. S. Bursi, R. Giannini, Problems and Perspectives in Seismic Quantitative Risk Analysis of Chemical Process Plants, Journal of Pressure Vessel Technology 141 (1) (2019) 010901.

[10] L. Di Sarno, G. Karagiannakis, Petrochemical Steel Pipe Rack: Critical Assessment of Existing Design Code Provisions and a Case Study, International Journal of Steel Structures 20 (1) (2020) 232-246.

[11] E. Salzano, I. Iervolino, G. Fabbrocino, Seismic risk of atmospheric storage tanks in the framework of quantitative risk analysis, Journal of Loss Prevention in the Process Industries 16 (5) (2003) 403-409.

[12] G. Fabbrocino, I. Iervolino, F. Orlando, E. Salzano, Quantitative risk analysis of oil storage facilities in seismic areas, Journal of Hazardous Materials 123 (1-3) (2005) 61-69.

[13] G. Lanzano, E. Salzano, F. S. de Magistris, G. Fabbrocino, Seismic vulnerability of natural gas pipelines, Reliability Engineering \& System Safety 117 (2013) 73-80. 
[14] G. Lanzano, F. Santucci de Magistris, G. Fabbrocino, E. Salzano, Seismic damage to pipelines in the framework of Na-Tech risk assessment, Journal of Loss Prevention in the Process Industries 33 (2015) 159-172.

[15] Task Committee on Seismic Evaluation and Design of Petrochemical Facilities of ASCE, Guidelines for Seismic Evaluation and Design of Petrochemical Facilities, second edition Edition, American Society of Civil Engineers, Reston, VA, 2011.

[16] H. N. Phan, F. Paolacci, O. S. Bursi, N. Tondini, Seismic fragility analysis of elevated steel storage tanks supported by reinforced concrete columns, Journal of Loss Prevention in the Process Industries 47 (2017) 57-65.

[17] O. S. Bursi, R. di Filippo, V. La Salandra, M. Pedot, M. S. Reza, Probabilistic seismic analysis of an LNG subplant, Journal of Loss Prevention in the Process Industries 53 (2018) 45-60.

[18] L. Di Sarno, G. Karagiannakis, On the seismic fragility of pipe rack—piping systems considering soil-structure interaction, Bulletin of Earthquake Engineering 18 (6) (2020) 2723-2757.

[19] D. Vamvatsikos, C. A. Cornell, Incremental dynamic analysis, Earthquake Engineering \& Structural Dynamics 31 (3) (2002) 491-514.

[20] K. Porter, R. Kennedy, R. Bachman, Creating Fragility Functions for Performance-Based Earthquake Engineering, Earthquake Spectra 23 (2) (2007) 471-489.

[21] J. W. Baker, Efficient Analytical Fragility Function Fitting Using Dynamic Structural Analysis, Earthquake Spectra 31 (1) (2015) 579-599.

[22] B. A. Bradley, A critical examination of seismic response uncertainty analysis in earthquake engineering, Earthquake Engineering \& Structural Dynamics 42 (11) (2013) 1717-1729.

[23] V. Silva, S. Akkar, J. Baker, P. Bazzurro, J. M. Castro, H. Crowley, M. Dolsek, C. Galasso, S. Lagomarsino, R. Monteiro, D. Perrone, K. Pitilakis, D. Vamvatsikos, Current Challenges and Future Trends in Analytical Fragility and Vulnerability Modeling, Earthquake Spectra 35 (4) (2019) 1927-1952.

[24] G. Abbiati, M. Broccardo, I. Abdallah, S. Marelli, F. Paolacci, Seismic fragility analysis based on artificial ground motions and surrogate modeling of validated structural simulators (2020). doi:10.31224/osf.io/bgjtf 
[25] S. Rezaeian, A. Der Kiureghian, A stochastic ground motion model with separable temporal and spectral nonstationarities, Earthquake Engineering \& Structural Dynamics 37 (13) (2008) 1565-1584

[26] A. H. Schellenberg, S. A. Mahin, G. L. Fenves, Advanced Implementation of Hybrid Simulation, Tech. Rep. PERR 2009/104, Pacific Earthquake Engineering Research (PEER) Center, University of California, Berkeley (2009).

[27] D. de Klerk, D. J. Rixen, S. N. Voormeeren, General Framework for Dynamic Substructuring: History, Review and Classification of Techniques, AIAA Journal 46 (5) (2008) 1169-1181.

[28] B. Sudret, Global sensitivity analysis using polynomial chaos expansions, Reliability Engineering \& System Safety 93 (7) (2008) 964-979.

[29] G. DeGrassi, Seismic Analysis of Large-Scale Piping Systems for the JNES-NUPEC Ultimate Strength Piping Test Program (NUREG/CR-6983), Tech. rep.

[30] O. S. Bursi, M. S. Reza, G. Abbiati, F. Paolacci, Performance-based earthquake evaluation of a full-scale petrochemical piping system, Journal of Loss Prevention in the Process Industries 33 (2015) 10-22.

[31] S. A. Karamanos, Mechanical Behavior of Steel Pipe Bends: An Overview, Journal of Pressure Vessel Technology 138 (4) (2016) 041203.

[32] P. K. Malhotra, T. Wenk, M. Wieland, Simple Procedure for Seismic Analysis of Liquid-Storage Tanks, Structural Engineering International 10 (3) (2000) 197-201.

[33] N. Mostaghel, Analytical Description of Pinching, Degrading Hysteretic Systems, Journal of Engineering Mechanics 125 (2) (1999) 216-224.

[34] S. Rezaeian, A. Der Kiureghian, Simulation of synthetic ground motions for specified earthquake and site characteristics, Earthquake Engineering \& Structural Dynamics (2010) n/a-n/a.

[35] B. Chiou, R. Darragh, N. Gregor, W. Silva, NGA Project Strong-Motion Database, Earthquake Spectra 24 (1) (2008) 23-44.

[36] J. W. Baker, Quantitative classification of near-fault ground motions using wavelet analysis, Bulletin of the Seismological Society of America 97 (5) (2007) 1486-1501. 
377 [37] G. Abbiati, I. Lanese, E. Cazzador, O. S. Bursi, A. Pavese, A computational framework for ${ }_{378}$ fast-time hybrid simulation based on partitioned time integration and state-space modeling, $379 \quad$ Structural Control and Health Monitoring 26 (10) (2019).

${ }_{380}$ [38] M. Berveiller, B. Sudret, M. Lemaire, Stochastic finite element: a non intrusive approach by regression, European Journal of Computational Mechanics 15 (1-3) (2006) 81-92.

[39] B. Liu, X. Liu, H. Zhang, Strain-based design criteria of pipelines, Journal of Loss Prevention in the Process Industries 22 (2009) 884-888.

[40] A. Suzuki, I. Iervolino, Intensity measure conversion of fragility curves, Earthquake Engineering \& Structural Dynamics 49 (6) (2020) 607-629. 